\title{
The effect of fast neutron irradiation on the performance of synthetic single crystal diamond particle detectors
}

\author{
A. Lohstroh $^{* 1}$, P. J. Sellin ${ }^{1}$, S. Gkoumas ${ }^{1}$, H. Al-Barakaty ${ }^{1}$, P. Veeramani ${ }^{1}$, M. E. Öszan ${ }^{1}$, G. \\ Prekas $^{1}$, M. C. Veale $^{1}$, J. M. Parkin ${ }^{1}$, and A.W. Davies ${ }^{1}$ \\ ${ }^{1}$ Department of Physics, University of Surrey, Guildford, GU2 7XH, UK
}

Diamond is known for its extreme hardness which may allow it to operate as a particle detector in high fluence environments even after absorption of large radiation doses. We present a study of the deterioration of the charge collection efficiency $(C C E)$ due to neutrons produced by ${ }^{235} \mathrm{U}$ fission, with irradiation fluences up to $1 \times 10^{16}$ $\mathrm{n} \mathrm{cm}^{-2}$. The planar devices were fabricated by thermal evaporation of Au onto approx. $300 \mu \mathrm{m}$ thick high purity chemical vapour deposited diamond produced by Element Six Ltd., UK. The detector performance was investigated as a function of bias voltage at room temperature using ${ }^{241} \mathrm{Am} \alpha$-particles and minimum ionising particles (MIPs) of a ${ }^{90} \mathrm{Sr}$ source. At low fluences up to $2 \times 10^{13}$ neutrons $\mathrm{cm}^{-2}$, the detectors reach the initial saturated signal amplitude after irradiation. However, the signal is less stable and deteriorates due to polarisation. This effect can be reduced by initial priming with X-rays. No peak could be distinguished in the detector response in the unprimed state after $10^{16}$ neutrons $\mathrm{cm}^{-2}$ with bias voltages up to. $1000 \mathrm{~V}$ (equivalent to $32 \mathrm{kV} \mathrm{cm}^{-1}$ ). However, a peak at about $18 \% C C E$ could be recovered after priming.

\section{Introduction}

\footnotetext{
* Corresponding author: e-mail: A.Lohstroh@surrey.ac.uk, Phone: +44 148368 9419, Fax: +44 1483686781
} 
Diamond has been of interest for radiation detection purposes in high radiation fluency environments due to its exceptional radiation hardness compared to other materials for many years. The effect of hadron induced radiation damage on the performance of particle detectors fabricated from polycrystalline diamond has been studied in detail with the particular goal to prove the devices' suitability and durability in applications for the Large Hadron collider (LHC). Up to $10^{16} \mathrm{n}_{\mathrm{eq}} \mathrm{cm}^{-2}$ are expected at $5 \mathrm{~cm}$ distance of the interaction point during 5 years in the super LHC [1]. In addition, neutron detection and damage studies are also of interest for the international fusion reactor ITER; where $10^{10} \mathrm{n} \mathrm{cm}^{-2} \mathrm{~s}^{-1}$, will be incident at the envisaged neutron detector position [2].

In the meantime, within less than the last decade, synthetic high purity chemical vapour deposited diamond with excellent electronic properties has been developed in several laboratories $[34,5,6]$ and become commercially available $[7,8]$. This has demonstrated diamonds large potential for electronic applications [9], especially for particle spectroscopy. Energy resolutions similar to well established Silicon devices has been achieved, and in addition to excellent timing properties [10]. Radiation damage studies on this high quality synthetic single crystal diamond material have begun to evaluate and gain understanding of the effects of radiation induced defects on the detection performance. $[2,11,12]$

The signal of a radiation detector operated as a solid state ionisation chamber is formed by the integrated induced current, i.e. the induced charge, at the electrodes of the detector by the movement of free charge carriers within the device towards those electrodes under the electric field they experience. The movement is limited by the charge carrier velocity and lifetime $\tau$ of the free charge carriers. In detector 
characterisation applications, the ratio of the created free charge carriers due to ionising radiation $Q_{0}$ and the "collected" induced charge $Q_{i}$ is commonly expressed as the charge collection efficiency $C C E=Q_{0} / Q_{i}$ [13]. Defects or defect complexes that act as charge trapping centres, will limit the lifetime of the free charge carriers, thus the $C C E$ and the detector performance, if they trap a significant proportion of the created charge carriers before they reach the device electrodes. In the particular case of diamond, which has a large band gap of $5.48 \mathrm{eV}$ [14], the charge trapped in deep levels can remain there over long periods of time (weeks) at room temperature, which alters the available empty trap level concentration and thus the carrier lifetime for subsequent irradiations, resulting in instabilities in the signal amplitude. The filling of deep levels results in an increase in carrier lifetime and thus signal amplitude. The effect is referred to as priming and is routinely used in the application of natural diamonds in medical dosimetry[15, 16]. However, the local filling of traps with charge carriers of one polarity will not just alter the free carrier lifetime, but also the electric field they experience due to the build up of space charge. This so called "polarisation" field is opposed to the direction of the applied electric field and reduces the charge carrier velocity, resulting in a drop of $C C E[17,18]$. Polarisation tends to be less pronounced if the ionising radiation penetrates through the whole device thickness [19] and can often easily be verified in diamond by the observation of signal pulses in the opposite polarity at zero bias after detector operation. If (some of) the defect levels present in the material responsible for priming and polarisation behaviour re-emit the trapped charge carriers within time scales that are comparable to the timescales in which the data is acquired, then the incident dose rate in addition to the total dose, will affect the detector performance as well. Reliable re-emission of trapped charge carriers in diamond is usually achieved by heating the samples above 
$550 \mathrm{~K}$ [20], but can also be induced by visible illumination [21]. The mechanism behind the latter is still not very well understood so far. It is clear that additional defects levels induced by radiation damage to the crystal structure will influence the performance and stability of diamond radiation detector due to the changes in carrier lifetime, polarisation and priming behaviour. Diamond is expected to be particular resilient to structural changes induced by radiation. It has a very rigid and tight lattice structure which leads to a large displacement energy of $32 \mathrm{eV}$ [22] for the carbon atoms bound in the lattice. The displacement of lattice atoms under irradiation will lead to the creation of carbon interstitials and vacancies. The mobility of these defects and subsequently their ability to move and form more complex defects or recombine depends on the temperature [23] and possibly on the defect structures (type and density) already present in the material. In addition to the polarisation and priming issues, these structural variations can compromise the comparability of radiation induced damage studies performed on different quality type samples from different sources. Furthermore, the radiation type introducing the damage also plays a role as discussed by de Boer and co-workers [12]. The charge carrier mobility may also be affected by radiation induced defects, although the results by Pomorski et al suggest that the changes in mobility -if present at all - are not significant up to a $26 \mathrm{MeV}$ proton dose of $10^{-14} \mathrm{~cm}^{-2}$ [19]. In this paper, we present the changes in particle detector performance and stability of a set of high purity synthetic single crystal chemical vapour deposited diamond samples due to neutron radiation induced damage to the bulk material.

\section{Materials and Methods}


We have studied four high purity single crystal diamond samples that were produced by Element Six, Ltd. by a chemical vapour deposition (CVD) process [3]. The samples have an area of $3 \times 3 \mathrm{~mm}^{2}$ each and the thickness varied between 306 and 309 $\mu \mathrm{m}$. They were boiled in a mixture of sulphuric acid and potassium nitrate by the supplier to provide high resistive, oxygen terminated surfaces. Subsequently, we cleaned the samples in aqua regia, followed by a rinse in acetone and isopropanol before thermally evaporating Au pad contacts on both sides of the nitrogen dried samples. Typical thicknesses of the gold layer varied between 30 and $70 \mathrm{~nm}$.

The dark current of the devices was measured with a computer controlled Keithley 487 picoammeter.

For the evaluation of the charge collection efficiency $(C C E)$, the devices were tested using the same set-up, electronics and calibration procedure as described by Lohstroh et al [21]. The samples are irradiated with non-collimated ${ }^{241} \mathrm{Am} \alpha$-particles (emission energy $5.48 \mathrm{MeV}$ ) in a vacuum chamber with a remaining pressure of less than $10^{-1}$ mbar. The emission spectrum of this source, studied with a conventional Silicon pin diode exhibits a broad peak with a centroid value of $4.95 \mathrm{MeV}$, most likely caused by absorption and straggling in the source material itself and in its thin protective coating. The source is mounted at about $8 \mathrm{~mm}$ distance to the sample and has an activity of $185 \mathrm{kBq}$. The $\alpha$-particle range in diamond is less than $15 \mu \mathrm{m}[24,25]$, which is a small fraction of the sample thickness; consequently, the induced charge signal is mainly due to hole movement under positive bias, and predominantly sensitive to electron movement at negative bias. The device response to minimum ionising particles was tested in a similar set-up. In this case, a higher gain charge sensitive pre-amplifier (eV product 550) was used and the samples were irradiated in 
air with $\beta^{-}$particles emitted from a $111 \mathrm{kBq}{ }^{90} \mathrm{Sr} /{ }^{90} \mathrm{Y}$ source. This isotope emits a wide range of electron energies. In order to only consider signals caused by high energy electrons $(>1.5 \mathrm{MeV})$ which are considered as MIPs, a Silicon surface barrier detector was mounted in transmission geometry to the diamond samples and used as a trigger. The spectra were calibrated in terms of deposited energy in the detector assuming that MIPs produce 36 free electron hole pairs per transversed $\mu \mathrm{m}$ in diamond [26]. The MIPs spectra were fitted using the so called Landau distribution in the form introduced by Moyal [27] given in equation (1). $A$ is a scaling parameter dependent on the absolute amplitude of the spectrum, i.e. the acquisition time; $\lambda=R\left(E_{m}-E\right)$, with $E_{m}$ being the most likely energy deposited in the sample and $\mathrm{R} \mathrm{a}$ material constant.

$$
P(\lambda)=A \frac{\exp [-0.5(\lambda+\exp (-\lambda))]}{\sqrt{2 \pi}}
$$

For priming studies, the samples were mounted in a light proof box and irradiated with $100 \mathrm{kVp}$ X-rays emitted by a Tungsten X-ray tube (Comet MXR225/22 powered by a Gulmay CP-225 supply). The absorbed does was 14 Gy in all cases and the samples were subsequently transferred into the vacuum chamber used for the $\alpha$ spectroscopy in a dark room.

\section{Table 1: Summary of the irradiation doses for each sample} Sample Thickness $[\mu \mathrm{m}] \quad$ Dose $\left[\mathrm{n} \mathrm{cm}^{-2}\right]$
A
308
$1 \times 10^{12}$
$\mathrm{B}$
309
$2 \times 10^{13}$ 
The gold contacts were removed using aqua regia before the neutron irradiations which were carried out by AWE at the VIPER facility. Three samples were irradiated with one to eleven $0.4 \mathrm{~ms}$ long neutron pulses emitted during ${ }^{235} \mathrm{U}$ fission with a broad energy spectrum that peaks at around $0.7 \mathrm{MeV}$. The received fluence was $1 \times 10^{12} \mathrm{n}$ $\mathrm{cm}^{-2}, 2 \times 10^{13} \mathrm{ncm}^{-2}$ and $1 \times 10^{16} \mathrm{n} \mathrm{cm}^{-2}$, for sample A, B, and $\mathrm{C}$ respectively, as summarised in table 1 . The samples were re-contacted as described above after the irradiation and the electrical characterisation repeated.

\section{Results and Discussion}

The initial detector response to $\alpha$-particles and MIPs has been studied before neutron irradiation. Each $\alpha$-particle spectrum was acquired for 10 minutes, and a summary of the extracted peak centroid positions in terms of $C C E$ as a function of the applied electric field strengths is shown in Fig.1.At low applied electric fields, weak polarisation effects were observed, which are attributed to the diamond/contact interface quality rather then the bulk material quality. These could also be responsible for the variation in performance of the different samples at low applied electric field strengths. Similar effects have been observed in the past [28] and are usually insignificant at higher applied bias. All samples exhibit a full energy peak at $100 \%$ $C C E$ above $3 \mathrm{kV} \mathrm{cm}^{-1}$. This confirms the suitability of these devices for spectroscopic applications if operated in the appropriate bias conditions.

After neutron irradiation, the response to $\alpha$-particle irradiation of the devices was investigated again. In contrast to sample B and C, the performance of sample A was 
effectively unchanged in the bias range investigated. The weak polarisation attributed to the diamond/contact interface was also observed in sample A after neutron irradiation and was not altered by the priming procedure. The response of sample B and $\mathrm{C}$ was found to be unstable and clearly affected more strongly by polarisation then before the neutron irradiation. The time evolution of the spectra was investigated by taking subsequent spectra for $90 \mathrm{~s}$ each under constant bias supply. An example of how the spectra change with time is given in the left part of Fig. 2. The change of the $C C E$ centroid position with time in sample A and B for the same applied field strength is illustrated in Fig 2 on the right hand side. Sample A exhibits a constant response at $100 \% C C E$ for both polarities, whereas the $C C E$ in sample B drops significantly during the first 10 minutes of irradiation, independent of the applied bias polarity. At the highest field strength for both polarities investigated $-32.4 \mathrm{kV} \mathrm{cm}^{-1}$ the centroid remained stable at about $95 \% C C E$ in sample B. No peak could be resolved in sample $\mathrm{C}$, which had received the highest neutron dose of this study of $10^{16} \mathrm{n} \mathrm{cm}^{-2}$; the absence of a peak under $\alpha$-particle irradiation in this sample in the non-primed state is illustrated in Fig. 3. For comparison, the first spectrum acquired of sample $\mathrm{C}$ at $32.4 \mathrm{kV} \mathrm{cm}^{-1}$ after priming is also shown in Fig.3, exhibiting a peak position of $18 \% C C E$. Subsequent spectra shift to lower amplitudes within 10 minutes and the same behaviour is found for the opposite polarity. Sample B reached maximum signal amplitude after priming at lower applied electric field strengths and the stability of the detector signal with time improved. Our results show that both electron and hole charge transport - are increasingly compromised by charge trapping and the subsequent build up of space charge that is characteristic polarisation processes, with increasing absorbed neutron dose. Further investigations, like transient induced current studies $[5,19,29]$ are needed to understand the effects of 
polarisation (i.e. changes in charge carrier velocity) and priming, (i.e. changes in charge carrier lifetime) on the observed changes in detector signals. Interestingly, improvements were found in both bias polarities, whereas it is assumed that in undamaged, polycrystalline diamond, priming mainly fills hole traps within the crystallites [30], charge transport remains limited by grain boundaries even in the primed state. Polarisation has also been observed in a $26 \mathrm{MeV}$ proton damage radiation study performed on diamond material of the same supplier [19]. The protons penetrated throughout the whole sample thickness and deposited energy responsible for the damage creation uniformly through the bulk. The authors of that work observe that space charge is not present initially after the damage has been introduced, but builds up during the subsequent characterisation, which is taken as an indication of the production of neutral defect states. This is also suggested by the findings of a thermally stimulated current damage study by Trajkov et al. [31]. The loss of a distinguishable peak in the spectrum fast neutron doses between $2 \times 10^{13} \mathrm{n} \mathrm{cm}^{-2}$ and $10^{16} \mathrm{n} \mathrm{cm}^{-2}$, agrees with the study by Tanaka et al, who observe a similar disappearance in a IIa high pressure high temperature synthesized samples with 14 $\mathrm{MeV}$ neutron irradiation above the dose of $5.5 \times 10^{13} \mathrm{n} \mathrm{cm}^{-2}[2]$. Various neutron reactions with ${ }^{12} \mathrm{C}$ are possible at energies up to $14 \mathrm{MeV}$ which subsequently lead to the emission of $\alpha$-particles as one of the reaction products [32] and deposit a larger amount of energy per unit path length in the detector than a neutron of equivalent energy.

The MIPs spectrum of sample B before neutron irradiation displayed in Fig.4 is representative of all non-irradiated samples studied. For comparison, the spectrum acquired from the same sample at the same applied effective field strength after 
neutron irradiation is also shown and exhibits essentially the same performance as in the virgin state. The polarisation phenomena observed so easily and clearly in the $\alpha$ particle spectra at the same applied field strength could not be studied on the same time scale of several minutes, due to the lower count rate in this experiment, caused by a number of reasons: only a small fraction of emitted $\beta$-particle is of sufficient energy to be a MIP, the lower activity of the source and the smaller solid angle determined by the experiments geometry. However, polarisation effects are also expected to be weaker because the MIPs penetrate and thus create electron hole pairs throughout the whole diamond thickness.

The MIP peaks could still be clearly separated from the noise edge in samples A. In contrast, up to the highest applied field strength explored in this study, $32.7 \mathrm{kV} \mathrm{cm}^{-1}$, no peak caused by MIPs could be separated from the noise edge in sample C. It shows higher counts in the presence of the radiation source - an indication that some sensitivity to MIPs has remained. Figure 5 summarises the fit results of the MIPs spectra. The most frequently detected signal energy $E_{m}$ converges towards the expected energy of $146 \mathrm{keV}$ with increasing bias voltages. The scaling parameter $A$ normalised to the acquisition time of each spectrum (not shown), fluctuates around a constant value for all spectra taken before irradiation and after, which firstly confirms the consistency of the fitting procedure and secondly indicated that the counting efficiency for the MIPs remained unchanged before and after the neutron irradiation up to $2 \times 10^{13} \mathrm{n} \mathrm{cm}^{-2}$. The extracted material constant $R$ for this high purity diamond also remained constant around a value of about $0.06 \mathrm{keV}^{-1}$.

\section{Conclusion}


We have fabricated simple sandwich structure radiation detectors based on high purity synthetic single crystal diamond and evaluated the degradation of its performance after fast neutron irradiation with doses up to $10^{16} \mathrm{n} \mathrm{cm}^{-2}$. Our results show that only at the highest level of irradiation, induced defects critically deteriorate the stability and reproducibility of the devices when operated as particle detector. This in agreement with other radiation induced damage studies on single crystal diamond detectors found in the literature. The signal amplitudes increases and the stability improves significantly by priming, however reliable operation in unknown irradiation fields is difficult to guarantee as all these effects depend on the incident absorbed dose and dose rate. In the case of MIPs that create electron hole pairs throughout the whole thickness of the studied devices, the performance before and after neutron irradiation up to $2 \times 10^{13} \mathrm{n} \mathrm{cm}^{-2}$ remained nearly unchanged at sufficiently high applied electric field strengths. However, at $10^{16} \mathrm{n} \mathrm{cm}^{-2}$ the signals could hardly be separated from the background.

The underlying correlation between polarisation and priming is not understood quantitatively so far. Further investigations separating charge carrier lifetime and velocity are needed as well as a more systematic study of the correlation between polarisation, priming and incident dose rate.

\section{Acknowledgments}

We are grateful for the collaboration with Element Six Ltd., the support of the AWE outreach program and Neville Bainbridge and the funding of the PPARC grant PP/D507607/1. The authors are also thankful to Gary Strudwick for his characterisation of the $\alpha$-source emission spectrum. 



\section{References}

[1] H. F. -W. Sadrozinski, Nucl. Instruments Methods A 552 (2005) 1-6.

[2] T. Tanaka, J. H. Kaneko, Y. Kasugai, M. Katagiri, H. Takeuchi, T. Nishitani, and

T. Iida, Diamond and Related Materials 14 (2005) 2031-2034

[3] J. Isberg, J. Hammersberg, E. Johansson, T. Wikström, D. J. Twitchen, A. J.

Whitehead, S. E. Coe, and G. A. Scarsbrook, Science 297 (2002) 1670-1672

[4] G. Bogdan, M. Nesladek, J. D’Haen, K. Haenen, and M. D’Olieslaeger, Diamond and Related Materials 15 (2006) 508-512

[5] N. Tranchant, D. Tromson, C. Descamps, A. Isambert, H. Hamrita, P. Bergonzo, and M. Nesladek, Diamond and Related Materials 17 (2008) 1297-1301

[6] M. Angelone, D. Lattanzi, M. Pillon, M. Marinelli, E. Milani, A. Tucciarone, G. Verona-Rinati, S. Popovichev, R. M. Montereali, M.A. Vincenti, A. Murari, and JETEFDA Contribtors, Nucl. Instruments Methods A 595 (2008) 616-622

[7] http://www.e6cvd.com (accessed 24th July 2009)

[8] http://www.diamonddetectors.com (accessed 24th July 2009)

[9] C. J. H. Wort, and R. S. Balmer, materialstoday 11(1-2) (2008) 22-28

[10] M. Pomorski, E. Berdermann, A. Caragheorgheopol, M. Ciobanu, M. Kišs, A. Martemiyanov, C. Nebel, and P. Moritz, for the NoRHDia Collabration, phys. stat. sol. (a) 203(12) (2006) 3152-3160.

[11] R. Wallny on behalf of the RD42 Collaboration, Nucl. Instrtuments Methods A $582(2007) 824-828$

[12] W. de Boer, J. Bol, A. Furgeri, S. Müller, C. Sander, E. Berdermann, M. Pomorski, and M. Huhtinen, phys. Stat. Sol. (a) 204(9) (2007) 3004-3010 
[13] G. F. Knoll, Radiation and Measurement, John Wiley \& Sons, Inc. New York (1999)

[14] A. Mainwood in "Properties and Growth of diamond" ed. by G. Davies, Inspec, London (UK) (1999) chapter 1.1, pp.3-8

[15] M. J. Guerrero, D. Tromson, M. Rebisz, C. Mer, B. Bazin, and P. Bergonzo, Diamond and Related Materials 13 (2004) 2046-2051

[16] A. Fidanzio, L. Azario, P. Viola, P. Ascarelli, E. Cappelli, G. Conte, and A. Piermattei, Nucl. Instruments and Methods A 524 (2004) 115-123

[17] E. Souw, and R. J. Meilunas, Nucl. Instruments Methods A (1997) 69-86

[18] C. Manfredotti, F. Fizzotti, P. Polesollo, and E. Vittone, Nucl. Instruments Methods A 426 (1999) 156-163

[19] M. Pomorksi, E. Berdermann, W. de Boer, A. Furgeri, C. Sander, and J. Morse for the NoRHDia Collaboration, Diamond and Related Materials 16 (2007) 10661068

[20] P. Bergonzo, D. Tromson, C.Descamps, H. Hamrita, C. Mer, N. Tranchant, and M. Nesladek, Diamond and Related Materials 15 (2007) 1038-1043

[21] A. Lohstroh, P. J. Sellin, S. G. Wang, A. W. Davies, and J. M. Parkin, Journal of Applied Physics 101 (2007) 063711

[22] D. W. Palmer in "Properties and Growth of diamond" ed. by G. Davies, Inspec, London (UK) (1999) chapter 5.1, pp.143-152

[23] S. Prawer, Diamond and Related Materials 4 (1995) 862-872

[24] http://www.srim.org

[25] J. Biersack, and L. Haggmark, Nucl. Instruments Methods 174 (1/2) (1980), 275

[26] W. Adam for the RD42 Collaboration, Nucl. Instruments Methods A 434 (1999) $131-145$ 
[27] R. J. Tapper, Rep. Prog. Phys. 63 (2000) 1273-1316

[28] A. Lohstroh, P. J. Sellin, S. Gkoumas, J. Parkin, P. Veeramani, G. Prekas, M. C. Veale, and J. Morse, phys. stat. sol. (a) 205(9) (2008) 2211-2215.

[29] S. Gkoumas, A. Lohstroh, and P. J. Sellin, Diamond and Related Materials (2009), doi:10.1016/j.diamond.2009.07.006

[30] M. Donato, G. Faggio, M. Marinelli, G. Messina, E. Milani, A. Paoletti, S. Santangelo, A. Tucciarone, G. Verona-Rinati, Diamond and Related Materials 10 (2001) 1788-1793

[31] E. Trajkov, S. Prawer, and P. Spizzirri, Diamond and Related Materials 12 (2003) 1738-1743

[32] M. Pillon, M. Angelone, and A. V. Krasilnikov, Nuclear Instruments and Methods B 101 (1995) 473 - 483 


\section{Figure Captions}

Fig 1: $C C E$ as a function of applied field strength before neutron irradiation Fig 2: Left: A series of pulse height spectra acquired for $90 \mathrm{~s}$ each. The $3^{\text {rd }}$ spectrum is omitted for clarity. Right: Peak position of the spectra illustrated in the left as a function of irradiation time. The horizontal error bars represent the duration $(90 \mathrm{~s})$ of each spectrums acquisition.

Fig 3: Pulse height spectrum of sample C after $10^{16} \mathrm{ncm}^{-2}$ irradiation in unprimed and primed condition.

Fig 4: Example of a MIPS spectrum before and after neutron irradiation of sample B

Fig 5: Summary of the fit parameters extracted from MIPS spectra of sample $\mathrm{A}$ and $\mathrm{B}$, before and after neutron irradiation 